\title{
A LEARNING THEOREM FOR LINEAR OPERATORS
}

\author{
JAN MYCIELSKI
}

(Communicated by R. Daniel Mauldin)

\begin{abstract}
We prove a convergence theorem in linear dynamic approximation theory which yields estimates of certain series of errors.
\end{abstract}

In [5 and 6] we have developed a model for the function of the cerebral cortex, which is based upon a theorem of linear dynamic approximation theory. Here we extend that theorem from the case of functionals to the case of operators. This allows new variants of the models developed in [6]. Instead of looking at the errors of each learning line separately, we can use now the errors of bunches of $n$ learning lines. But the theorem which is proved below has a straightforward motivation which does not require the knowledge of $[5,6]$. A sequence of points $\xi_{0}, \xi_{1}, \ldots \in \mathbf{R}^{k}$ represent the consecutive inputs to a system $S$. Other inputs $y_{0}, y_{1}, \ldots \in \mathbf{R}^{n}$ represent the desired outputs of $S$ but they reach $S$ a little later so that $S$ must compute its guess $\hat{y}_{t} \in \mathbf{R}^{n}$ on the basis of $\xi_{0}, \ldots, \xi_{t}$ and $y_{0}, \ldots, y_{t-1}$, before getting $y_{t}$. Of course $y_{t}-\hat{y}_{t}$ is the error vector and this error is known to $S$ at the time when $\hat{y}_{t+1}$ is to be computed. We want to design $S$ such that the errors will be minimized in some sense, although this will require of course certain assumptions about the sequences $\left(\xi_{t}\right)$ and $\left(y_{t}\right)$. (In our model in [6] the neocortex is represented as a system of millions of overlapping thin columns perpendicular to the cortical layers. Each such column contains a system $S$. At the bottom of each of them the inputs $\xi_{t}$ and $y_{t}$ are received and in that particular model $y_{t}$ is one of the coordinates of $\xi_{t+1}$.)

We specify $S$ as follows. First $S$ has a fixed (nonlinear) preparatory map $\phi: \mathbf{R}^{k} \rightarrow \mathbf{R}^{m}-\{0\}$. For example, $\phi$ could be the system of all monomials of degree $\leq d$ in the coordinates of $\mathbf{R}^{k}$. (In the applications in [6] there is a brief discussion of the $\phi$ 's which appear natural for the model of the neocortex considered there. In that case surely $d \leq 3$ but still $k$ is too large so that not all $\left(\begin{array}{c}k+3 \\ 3\end{array}\right)$ monomials of degree $\leq 3$ could appear in $\phi$. On the other hand functions of a different nature, such as characteristic functions of intervals, could appear among the coordinates of $\phi$.) This map $\phi$ does not depend on $t$. Then $S$ seeks a linear map $M_{t}: \mathbf{R}^{m} \rightarrow \mathbf{R}^{n}$ such that the error vector $y_{t}-M_{t}\left(\phi\left(\xi_{t}\right)\right)$ be small in a sense. So in our system $S$, we have $\hat{y}_{t}=M_{t}\left(\phi\left(\xi_{t}\right)\right)$. The linear operator $M_{t}$ is called the long term memory of $S$ while $\phi$ could be called the instinctive memory of $S . M_{t}$ is computed from $\phi\left(\xi_{t-1}\right), M_{t-1}$ and $y_{t-1}$ only, and this computation is very simple so that it can be accomplished in fractions of a second by the tissues of the central nervous system.

Received by the editors December 20, 1986.

1980 Mathematics Subject (lassification (1985 Revision). Primary 41A25; Secondary 92A08, $92 \mathrm{~A} 25$. 
In the present paper $\phi$ will play no role. So we put $x_{t}=\phi\left(\xi_{t}\right)$ and we study only a subsystem $A$ of $S$ which is defined as follows.

$t=0,1,2, \ldots$ is the time variable.

$x_{t} \in \mathbf{R}^{m}-\{0\}$ is the input to $A$ at time $t$.

$y_{t} \in \mathbf{R}^{n}$ is the desired output of $A$ at time $t$.

Both $x_{t}$ and $y_{t}$ are viewed as column vectors.

$L_{m n}$ is the space of linear maps $M: \mathbf{R}^{m} \rightarrow \mathbf{R}^{n}$, i.e., of real $(m \times n)$-matrices.

$M_{t} \in L_{m n}$ is the state of the memory of $A$ at time $t$.

$\hat{y}_{t}=M_{t} x_{t}$ is the prediction of $y_{t}$ which $A$ makes at time $t$.

The error of this prediction is defined by the formula

$$
e_{t}=\left(y_{t}-\hat{y}_{t}\right) /\left\|x_{t}\right\| \text {. }
$$

So $e_{t} \in \mathbf{R}^{n}$ and $e_{t}$ is a vector of relative errors.

Given any $M_{0} \in L_{m n}$, the memory of $A$ at time $0, A$ updates its memory according to the following formula

$$
M_{t+1}=M_{t}+\left[1-\frac{\theta}{\left\|e_{t}\right\|}\right]_{+} \frac{e_{t} x_{t}^{T}}{\left\|x_{t}\right\|},
$$

where $\theta \geq 0$ is a certain constant, $[\alpha]_{+}=\max (0, \alpha)$ and $x_{t}^{T}$ is the transpose of $x_{t}$.

It is easy to check that the formula (1) is equivalent to the following statement. $M_{t+1}$ is the operator which minimizes the norm $\left\|M_{t+1}-M_{t}\right\|$ while satisfying the inequality

$$
\left\|y_{t}-M_{t+1} x_{t}\right\| /\left\|x_{t}\right\| \leq \theta \text {. }
$$

(Recall the definition: for all $M=\left(a_{i j}\right) \in L_{m n}$,

$$
\left.\|M\|=\left(\sum_{i j} a_{i j}^{2}\right)^{1 / 2}=\left(\operatorname{Tr}\left(M M^{T}\right)\right)^{1 / 2} .\right)
$$

The rule (1) is motivated by its computational simplicity and the upper bounds given in the theorem below.

We still need the notations

$$
e_{t}(M)=\left(y_{t}-M x_{t}\right) /\left\|x_{t}\right\|
$$

in particular $e_{t}=e_{t}\left(M_{t}\right)$,

$$
E_{s}(M)=\operatorname{Sup}_{t \geq s}\left\|e_{t}(M)\right\|
$$

and, for $\alpha=1,2$, we define the following series of errors

$$
S_{\alpha}=\sum_{t=0}^{\infty}\left(\left[\left\|e_{t}\right\|-\theta\right]_{+}\right)^{\alpha} .
$$

With these notations our result takes the following form

THEOREM. (i) $\left\|M_{t+1}-M_{t}\right\|=\left[\left\|e_{t}\right\|-\theta\right]_{+}$.

(ii) $S_{2} \leq \operatorname{Inf}\left\{\left\|M-M_{0}\right\|^{2} \mid E_{0}(M) \leq \theta\right\}$.

(iii) $S_{1} \leq \operatorname{Inf}\left\{\left(\left\|M-M_{0}\right\|^{2}-S_{2}\right) / 2\left(\theta-E_{0}(M)\right) \mid E_{0}(M)<\theta\right\}$. 
(iv) If $\operatorname{Inf}\left\{E_{s}(M) \mid M \in L_{m n}, s=0,1, \ldots\right\}<\theta$, then $S_{1}<\infty$.

(We have stipulated here that $\operatorname{Inf} \varnothing=\infty$.)

ProOF. (i) is obvious from (1).

To show (ii) we define $x_{t}^{0}=x_{t} /\left\|x_{t}\right\|, \sigma_{t}=\left\|M-M_{t}\right\|^{2}$ and $\alpha_{t}=\left[1-\theta /\left\|e_{t}\right\|\right]_{+}$. By (2) we have

$$
\left(M-M_{t}\right) x_{t}^{0}=e_{t}-e_{t}(M)
$$

and, by (3),

$$
\left\|e_{t}(M)\right\| \leq E_{0}(M)
$$

A routine calculation using (4), Schwarz's inequality and (5) yields

$$
\begin{aligned}
\sigma_{t+1} & =\left\|\left(M-M_{t}\right)-\alpha_{t} e_{t} x_{t}^{0 T}\right\|^{2} \\
& =\sigma_{t}-2 \alpha_{t}\left\|e_{t}\right\|^{2}+2 \alpha_{t} \operatorname{Tr}\left(e_{t}(M) e_{t}^{T}\right)+\alpha_{t}^{2}\left\|e_{t}\right\|^{2} \\
& \leq \sigma_{t}-2 \alpha_{t}\left\|e_{t}\right\|^{2}+2 \alpha_{t} E_{0}(M)\left\|e_{t}\right\|+\alpha_{t}^{2}\left\|e_{t}\right\|^{2} \\
& =\sigma_{t}-\left[\left\|e_{t}\right\|-\theta\right]_{+}^{2}-2\left(\theta-E_{0}(M)\right)\left[\left\|e_{t}\right\|-\theta\right]_{+} .
\end{aligned}
$$

Hence, since $\sigma_{t+1} \geq 0$ for all $t$, we get

$$
0 \leq \sigma_{0}-S_{2}-2\left(\theta-E_{0}(M)\right) S_{1} .
$$

So if $\exists M\left[\theta \geq E_{0}(M)\right]$ we get (ii) and if $\exists M\left[\theta>E_{0}(M)\right]$ we get (iii).

(iv) follows of course from (3) and (iii).

REMARKS. 1. The work of Gersho and Lucky $[\mathbf{1}, \mathbf{2}, \mathbf{3}, \mathbf{4}]$ (see also $[\mathbf{7}, \mathbf{8}]$ and references therein) suggests that if the inputs $x_{t}$ are loaded with random noise, then the rule (1) should be replaced by the rule

$$
M_{t+1}=M_{t}+c\left[1-\frac{\theta}{\left\|e_{t}\right\|}\right]_{+} \frac{e_{t} x_{t}^{T}}{\left\|x_{t}\right\|}
$$

where $c$ is a suitable constant in the interval $[0,1]$. But we do not know any clear-cut theorem elucidating this situation.

2. One can replace the domain space $\mathbf{R}^{m}$ by any Hilbert space and it is easy to generalize the theorem accordingly. One can replace both spaces $\mathbf{R}^{m}$ and $\mathbf{R}^{n}$ by the Hilbert space $l_{2}$, but then our norm $\left\|\left(a_{i j}\right)\right\|=\left(\sum a_{i j}^{2}\right)^{1 / 2}$ may be infinite. So our Theorem may be significant only for appropriate choices of $M_{0}$. The unit matrix is a natural choice for $M_{0}$ in such applications in which $y_{t}=x_{t+1}$, for $t=0,1, \ldots$

3 . One can also modify the theorem to a continuous time variable in the style of $\S 3$ in [6].

\section{REFERENCES}

1. A. Gersho, Automatic equalization technique for multilevel pulse transmissions, Bell Telephone Laboratories Technical Memorandum, MM 65-1381-13, December 1965.

2. __ Adaptive equalization of highly dispersive channels for data transmission. I, Bell Telephone Laboratories Technical Memorandum, MM 68-1386-3, April 1968.

3. R. W. Lucky, Automatic equalization for digital communication, Bell System Tech. J. 44 (1965), 547-588.

4. __ Techniques for adaptive equalizations for digital communication systems, Bell System Tech. J. 45 (1966), 255-286. 
5. J. Mycielski, Can mathematics explain natural intelligence?, Physica 22D (1986), 366-375. Also appeared as a Los Alamos National Laboratory Report LA-10492-MS, UC32 July 1985.

6. J. Mycielski and S. Swierczkowski, A model of the neocortex, Los Alamos National Laboratory Report LA-11140-MS, UC32 November 1987.

7. R. S. Sutton, Learning to predict by the method of temporal differences, Technical Report TR87509.1, GTE Laboratories Inc., Waltham, Mass., 1987.

8. B. Widrow and S. D. Stearns, Adaptive signal processing, Prentice-Hall, Englewood Cliffs, N. J., 1985.

DEPARTMENT OF MATHEMATICS, UNIVERSITY OF COLORADO, BOULDER, COLORADO 80309-0426 